\section{Reduction of exposure to plasticizers in stored red blood cell units}

\author{
Frank Münch, ${ }^{1}$ Thomas Göen, ${ }^{2}$ Robert Zimmermann, ${ }^{3}$ \\ Werner Adler, ${ }^{4}$ Ariawan Purbojo,' Christine Höllerer, ${ }^{2}$ \\ Robert Anton Cesnjevar' and André Rüffer'
}

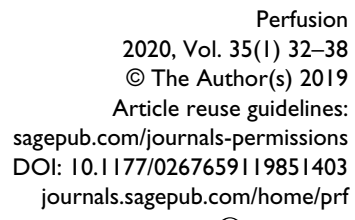

@SAGE

\begin{abstract}
Introduction: Plastic can be toxic and hazardous to an organism's health, but it is being widely used in our daily lives. Di2-ethylhexyl-phthalate is the most common plasticizer in medical devices made of polyvinylchloride and is commonly found in soft bags storing red blood cell units. Di-2-ethylhexyl-phthalate and its degradation product mono-2-ethylhexylphthalate can migrate into human body fluids, for example, blood and tissues. The aim of the study was to assess the concentration of plasticizers in red blood cell units according to storage time and after mechanical rinsing using a cell salvage device.

Methods: Levels of di-2-ethylhexyl-phthalate and mono-2-ethylhexyl-phthalate were analysed in 50 unwashed red blood cell units using liquid chromatography coupled with tandem mass spectrometry. In addition, phthalate concentrations were measured before and after mechanical rinsing in six more washed red blood cell units with storage times ranging between 36 and 56 days. A linear regression model was determined by the daily increase of di-2-ethylhexyl-phthalate and mono-2-ethylhexyl-phthalate in the stored red blood cell units subject to their storage time (range $=4-38$ days), and the effect of mechanical rinsing on their phthalate concentration was calculated.

Results: A linear correlation was found between storage time of unwashed red blood cell units and the concentration of di-2-ethylhexyl-phthalate $(p<0.00 \mathrm{I})$ or mono-2-ethylhexyl-phthalate $(p<0.00 \mathrm{I})$. Stored red blood cell units older than 14 days had significantly higher concentrations of both contaminants than red blood cell units of shorter storage time $(p<0.001)$. Mechanical rinsing in washed red blood cell units attained a reduction in the di-2-ethylhexyl-phthalate and mono-2-ethylhexyl-phthalate concentration by a median of $53 \%($ range $=18-68 \% ; p=0.031$ ) and $87 \%($ range $=68-96 \%$; $\mathrm{p}=0.03 \mathrm{I})$, respectively.

Conclusion: Leaching of di-2-ethylhexyl-phthalate and mono-2-ethylhexyl-phthalate into red blood cell units depends on the duration of storage time. Plasticizers can be significantly reduced by mechanical rinsing using cell salvage devices, and thus, red blood cell units can be regenerated with respect to chemical contamination.
\end{abstract}

\title{
Keywords
}

plasticizers; red blood cell units; mechanical rinsing; storage time; di-2-ethylhexyl-phthalate

\section{Introduction}

Plastic is made flexible by the addition of plasticizers. Di-2-ethylhexyl-phthalate (DEHP) is the most prevalent synthetic polymer softener in medical devices and

\footnotetext{
'Department of Pediatric Cardiac Surgery, University Hospital Erlangen, Friedrich-Alexander-Universität Erlangen-Nürnberg (FAU), Erlangen, Germany

2Institute and Outpatient Clinic of Occupational, Social and Environmental Medicine, University Hospital Erlangen, FriedrichAlexander-Universität Erlangen-Nürnberg (FAU), Erlangen, Germany ${ }^{3}$ Department of Transfusion Medicine and Haemostaseology, University Hospital Erlangen, Friedrich-Alexander-Universität Erlangen-Nürnberg (FAU), Erlangen, Germany
}

is almost exclusively used in stored bags for red blood cell units (RBCUs). Particularly during invasive treatment, when used to deliver enteral or parenteral lipophilic fluids (i.e. blood), leaching of DEHP and its degradation product mono-2-ethylhexyl-phthalate

${ }^{4}$ Institute of Medical Informatics, Biometry and Epidemiology, University Hospital Erlangen, Friedrich-Alexander-Universität Erlangen-Nürnberg (FAU), Erlangen, Germany

\section{Corresponding author:}

Frank Münch, Department of Pediatric Cardiac Surgery, University Hospital Erlangen, Friedrich-Alexander-Universität Erlangen-Nürnberg (FAU), Loschgestrasse 15, 91054 Erlangen, Germany.

Email: frank.muench@uk-erlangen.de 
(MEHP) into the recipient's circulation and its deposition in human tissue have been frequently attested. ${ }^{1-8}$

In the last decades, considerable concerns regarding the side effects of plasticizers on endocrine, testicular, ovarian and neural function, as well as their hepatotoxic and cardiotoxic impact have been raised..$^{9-11}$ The distribution of phthalates and other plasticizers is being regulated by the European Union (EU). ${ }^{12}$ Surprisingly, although DEHP and five other phthalates are now forbidden in the manufacturing of children's toys, the use of phthalates in medical products is still permissible. ${ }^{13}$

Cell salvage devices are used during surgical procedures in order to collect and recycle autologous blood. Collected blood components are separated into its constituents according to their weight by centrifugal force and washed with a $0.9 \%$ saline solution. Erythrocytes are pressed to the outer wall of the preparation bowl, and with increasing centrifugal speed, the low mass blood components are aspirated in the middle. The elimination rate of anticoagulation solutions, free haemoglobin and potentially dangerous substances ranges from $75 \%$ to over $90 \% .^{14,15}$ This results in a purified product of concentrated autologous erythrocytes suspended in normal saline. However, to date, it is unknown in which way plasticizers in RBCUs can be diminished by mechanical rinsing using a cell salvage device.

The aim of the study was to measure the content of DEHP and MEHP in stored unwashed RBCUs and to explore the reduction of these contaminants in washed RBCUs by cell salvage application.

\section{Methods}

\section{Storage conditions for RBCUs}

A total of 56 leucocytes-reduced RBCUs, preserved in saline-adenine-glucose-mannitol solution (SAG-M), were obtained from the Institute for Transfusion Medicine (Suhl, Germany). All units were handled according to German guidelines for the collection of blood and blood components. ${ }^{16}$ The manufacturer performed leucocytes reduction of whole blood or buffy coat-reduced RBCUs after separation into components by inline filtration on the collection day, or the day after, at the latest 22 hours after donation. All RBCUs were stored at $4^{\circ} \mathrm{C}$ immediately after preparation. On Day 2 or Day 3 after collection, all RBCUs were shipped to our institution. Thereafter, RBCUs were stored at $+4^{\circ} \mathrm{C}\left( \pm 2^{\circ} \mathrm{C}\right)$ in a refrigerator (Kirsch, Offenburg, Germany). Cold storage was only interrupted to obtain samples for cross-matching.

\section{Plasticizers in unwashed RBCUs}

In total, 50 stored RBCUs were analysed before being transfused to patients. Storage times for application of each RBCU were documented in the study protocol. Blood samples $(5 \mathrm{~mL})$ were stored at $-20^{\circ} \mathrm{C}$ in glass vials. Stored blood samples were analysed for the plasticizers DEHP and MEHP. The analytical determination was performed using liquid chromatography coupled with tandem mass spectrometry according to a previously published analytical method. ${ }^{17,18}$ The limits of quantitation (LOQ) for DEHP and MEHP were 5 and $2 \mu \mathrm{g} / \mathrm{L}$, respectively. The content of DEHP and MEHP in RBCUs was determined depending on storage time.

\section{Plasticizers in washed RBCUs}

Six additional stored RBCUs were intentionally chosen according to the longest storage time (median $=37$ days, range $=36-56$ days) in order to obtain RBCUs with higher levels of DEHP and MEHP. The recovery and storage of the RBCUs is similar to the above guidelines. ${ }^{16}$ A standard cell salvage machine (Elekta; Sorin Group ${ }^{\odot}$ ) fitted with a 175-mL washing bowl was filled with $300 \mathrm{~mL}$ of donor blood (RBC) at a rate of $250 \mathrm{~mL} / \mathrm{min}$ centrifugal speed. ${ }^{15}$ After filling the bowl, the rinsing programme started automatically using $600 \mathrm{~mL}$ of physiological saline solution ( $\mathrm{NaCl} 0.9 \%$, 'phtalate-free') and a pump rotation speed of $350 \mathrm{~mL} / \mathrm{min}$. The 'washed RBCUs' were transferred into a new plastic bag at a rate of $200 \mathrm{~mL} / \mathrm{min}$ pump rotational speed. Blood samples $(5 \mathrm{~mL})$ were taken before and after mechanical rinsing, respectively, and stored in glass vials until analysis. Accordingly, levels of DEHP and MEHP were measured as stated above.

\section{Theoretical model to illustrate patient stress}

A statistical linear regression model was determined by the daily increase of DEHP and MEHP in unwashed RBCUs. Accordingly, phthalate concentrations were calculated according to the storage time. Important toxic outcomes of DEHP also resulted from adverse effects of its degradation product MEHP, which stresses a joint assessment of both compounds. Therefore, to assess the total burden of plasticizers in RBCUs, the DEHP equivalent $\left(\mathrm{DEHP}_{\mathrm{eq}}\right)$ calculation is needed due to the different molecular masses of DEHP and MEHP. Molecular weights $(\mathrm{DEHP}=390.56 \mathrm{~g} / \mathrm{mol}$ and $\mathrm{MEHP}=278.35 \mathrm{~g} / \mathrm{mol})$ were set in proportion to obtain the conversion factor

$$
\frac{390.56 \frac{\mathrm{g}}{\mathrm{mol}}}{278.35 \frac{\mathrm{g}}{\mathrm{mol}}}=1.403
$$

To calculate $\mathrm{DEHP}_{\text {eq }}$, the following formula was used

$$
\operatorname{DEHP}_{\mathrm{eq}}=\operatorname{DEHP}[\mathrm{mg} / \mathrm{L}]+1.403 \operatorname{MEHP}[\mathrm{mg} / \mathrm{L}]
$$




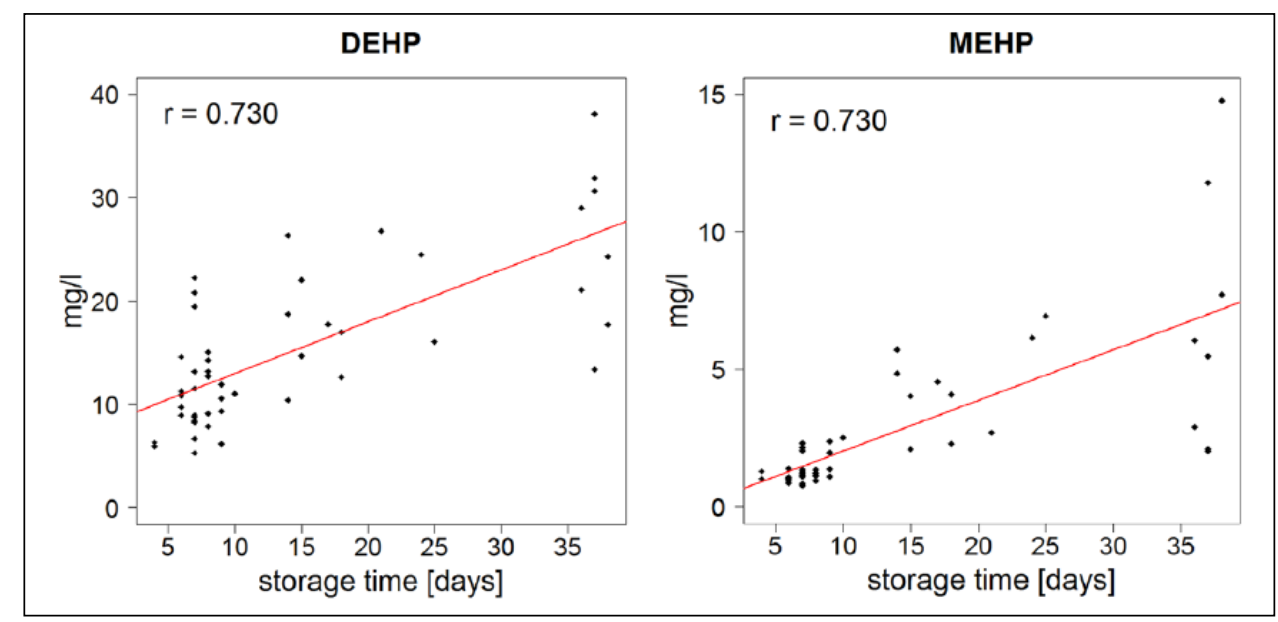

Figure I. Linear regression between storage time of 50 RBCUs and DEHP or MEHP concentrations.

The tolerable daily intake (TDI) level indicates the amount of the daily intake of a product at which no adverse effects on health have to be expected. For safety, TDI levels are set by dividing the "No Observed Adverse Effect Level' found in animal studies with an adjustment factor of 100. In case of DEHP, the TDI is $50 \mathrm{mg} / \mathrm{kg}$ body weight. 919,20

Patient stress by phthalate contamination and the effect of mechanical rinsing were calculated for a $5-\mathrm{kg}$ child. Calculations included the following:

1. The content and exposure to $\mathrm{DEHP}_{\text {eq }}$ after administration of 100-mL unwashed RBCUs.

2. The calculated total burden of plasticizers $\left(\mathrm{DEHP}_{\mathrm{eq}}\right)$ was related (\%) to the TDI of DEHP. The allowed TDI of DEHP $(0.05 \mathrm{mg} / \mathrm{kg}$ body weight) in a $5-\mathrm{kg}$ child is $0.25 \mathrm{mg}$.

3. The effect of mechanical rinsing on reducing the total burden of plasticizers was calculated after administration of $100 \mathrm{~mL}$ of washed RBCUs when compared to unwashed RBCUs.

\section{Statistics}

The data are expressed as mean \pm standard deviation (SD) with $95 \%$ confidence interval (95\% CI). The correlation between storage time (days) of 50 RBCUs and their content of DEHP and MEHP was calculated using Pearson's correlation method. To identify a threshold of storage time, we divided the RBCUs into two groups depending on their content of plasticizers. In the following, two conditional inference trees ${ }^{21}$ using depth one (only one branch) were modelled with regard to time, MEHP and DEHP as independent variables. Threshold values were determined by the trees as cut-off points. Significance between groups was calculated by the nonparametric Mann-Whitney $\mathrm{U}$ test or $t$-test, according to distribution. To examine the difference between content of DEHP and MEHP in RBCUs before and after mechanical rinsing, a Wilcoxon signed-rank test was used. A p-value of $<0.05$ was considered statistically significant. All statistical analyses were conducted using the software R version 3.3.0 (R Foundation for Statistical Computing).

\section{Results}

\section{Unwashed RBCUs}

The 50 RBCUs were received from the blood bank between 4 and 38 days after preparation. Median storage time of RBCUs was 8 days. The DEHP and MEHP levels in the RBCUs ranged between $5.3-38.1 \mathrm{mg} / \mathrm{L}($ median $=13.0 \mathrm{mg} / \mathrm{L})$ and $0.8-14.8 \mathrm{mg} / \mathrm{L}$ (median $=1.4 \mathrm{mg} / \mathrm{L}$ ), respectively. A significant correlation was found between storage time of RBC and the DEHP (Pearson $=0.730 ; \mathrm{p}<0.001$ ) and MEHP $($ Pearson $=0.730 ; \mathrm{p}<0.001)$ concentrations (Figure 1).

RBCUs stored for less than 14 days had significantly lower DEHP and MEHP concentrations than RBCUs stored for a longer time $(p<0.001)$. In RBCUs stored for less than 14 days $(\mathrm{n}=31 /$ median $=7$ days), DEHP and MEHP levels were $11.0 \pm 4.2 \mathrm{mg} / \mathrm{L}(95 \% \mathrm{CI}=9.6,12.5)$ and $1.3 \pm 0.5 \mathrm{mg} / \mathrm{L}(95 \% \mathrm{CI}=1.2,1.5)$, respectively. In RBCUs stored for $\geqslant 14$ days $(n=19 /$ median $=24$ days $)$, DEHP and MEHP levels were $21.7 \pm 7.4 \mathrm{mg} / \mathrm{L}(95 \%$ $\mathrm{CI}=18.4,25.1)$ and $5.3 \pm 3.4 \mathrm{mg} / \mathrm{L}(95 \% \mathrm{CI}=3.7,6.9)$, respectively (Figure 2 ).

\section{Washed RBCUs}

In RBCUs used for cell salvage processing $(n=6)$, the average storage time was $31.2 \pm 8.8$ days. After the mechanical rinsing through the cell salvage device, levels of DEHP and MEHP were found to be significantly 




Figure 2. DEHP and MEHP release into 31 RBCUs stored for $<14$ days versus 19 RBCUs stored for $\geqslant 14$ days.

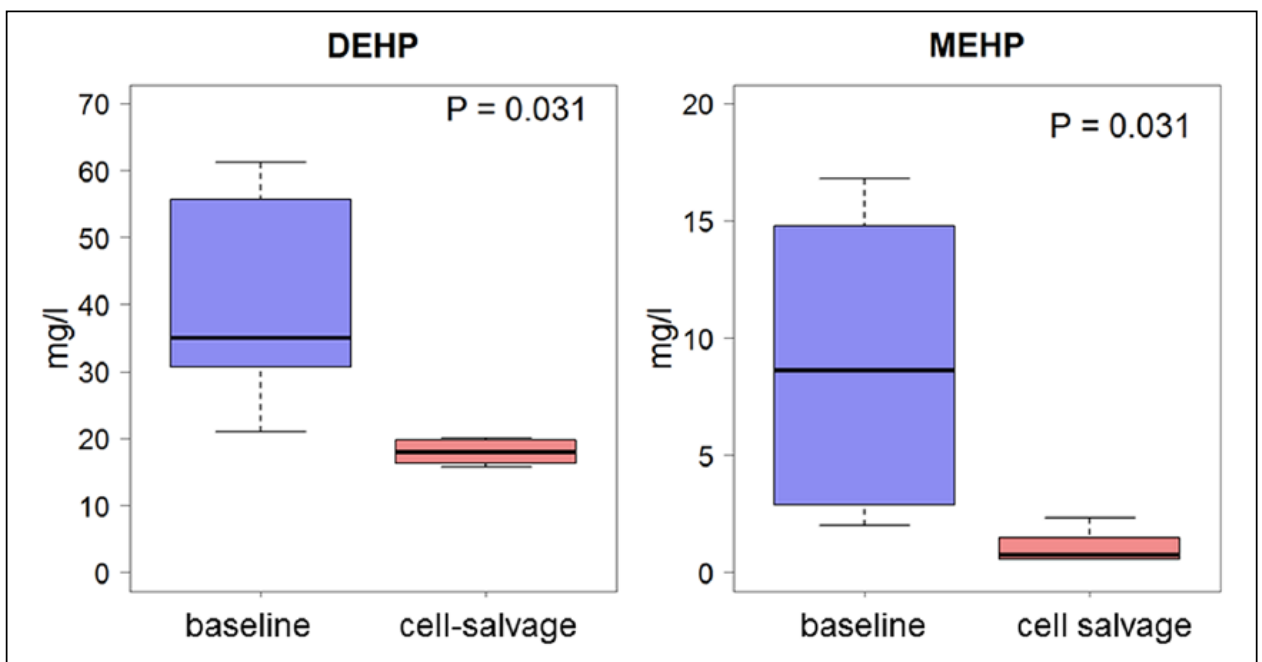

Figure 3. Reduction of DEHP and MEHP before and after mechanical rinsing of six RBCUs.

lower when compared to baseline levels (DEHP at baseline $=40 \pm 14 \mathrm{mg} / \mathrm{L}(95 \% \mathrm{CI}=27,52)$ versus DEHP after mechanical rinsing $=18 \pm 2 \mathrm{mg} / \mathrm{L} \quad(95 \% \mathrm{CI}=17,19)$; $\mathrm{p}=0.031$ and MEHP at baseline $=9 \pm 6 \mathrm{mg} / \mathrm{L}(95 \%$ $\mathrm{CI}=4$, 14) versus MEHP after mechanical rinsing $=1 \pm 0.7 \mathrm{mg} / \mathrm{L}(95 \% \mathrm{CI}=0.5,1.6) ; \mathrm{p}=0.031)$. Thus, cell salvage processing attained a reduction in the DEHP and MEHP content by a median of $53 \%$ (range $=18-68 \%$; $\mathrm{p}=0.031)$ and $87 \%$ (range $=68-96 \% ; \mathrm{p}=0.031$ ), respectively (Figure 3).

\section{Theoretical model}

The TDI level in a 5-kg child was overcome three-fold after administration of $100-\mathrm{mL}$ untreated RBCUs at Day 1 of storing and was increased 14.8-fold after
38 days of storing. In washed RBCUs, the TDI from DEHP $_{\text {eq }}$ would be reduced by 1.5-5.6 according to storage time (Table 1).

\section{Discussion}

All tested RBCUs used in this study contained the plasticizer DEHP as well as its degradation product MEHP. Levels of both compounds increased significantly when the storage time was longer, particularly after 14 days. A phthalate contamination of RBCUs, as well as its association with storage time, has been previously described by other groups. ${ }^{7,22,23}$ Compared with these, phthalate levels found in this study were somewhat lower than reported, which may indicate an improvement of product quality during the last decade. Nevertheless, the 
Table I. Calculated content and exposure to plasticizers after administration of $100-\mathrm{mL}$ unwashed and washed RBCUs in a 5-kg child.

\begin{tabular}{|c|c|c|c|c|c|}
\hline Storage time (days) & I & 7 & 14 & 28 & 38 \\
\hline \multicolumn{6}{|l|}{ Unwashed RBCUs } \\
\hline $\mathrm{DEHP}_{\mathrm{eq}}(\mathrm{mg} / \mathrm{l} 00 \mathrm{~mL})$ & 0.82 & 1.4 & 1.9 & 3.0 & 3.7 \\
\hline Increase of TDI ${ }^{\mathrm{a}}(\%)$ & 330 & 540 & 760 & 1,180 & 1,480 \\
\hline \multicolumn{6}{|l|}{ Washed RBCUs } \\
\hline $\mathrm{DEHP}_{\text {eq }}(\mathrm{mg} / \mathrm{I} 00 \mathrm{~mL})$ & 0.38 & 0.57 & 0.75 & 1.13 & 14.0 \\
\hline Reduction of DEHP $\mathrm{eq}$ by mechanical rinsing (\%) & 54 & 58 & 60 & 62 & 62 \\
\hline Reduction of TDI by mechanical rinsinga (\%) & 150 & 230 & 300 & 450 & 560 \\
\hline
\end{tabular}

RBCUs: red blood cell units; DEHP: di-2-ethylhexyl-phthalate; TDI: tolerable daily intake.

aAllowed TDI of DEHP $(0.05 \mathrm{mg} / \mathrm{kg}$ body weight $)$ in a $5-\mathrm{kg}$ child is $0.25 \mathrm{mg}$.

persistent exceedance of the TDI for DEHP may be still a cause of concern for health effects and should be avoided if alternative solutions exist.

Storage of RBCUs is associated with an almost complete loss of 2,3-diphosphoglycerate within 2 weeks and increasing signs of cell damage, for example, cell degradation, changes in electrolyte balance, particularly the release of potassium, increased levels of lactate and citrate and deformation of erythrocytes. Particularly in infants and toddlers, storage time of RBCUs should be kept short in order to reduce the exposure of erythrocytes to phthalates. ${ }^{24}$

Mechanical rinsing of stored blood aliquots is commonly used prior transfusion in order to clean them from exceeding electrolyte imbalances. ${ }^{24}$ A reduction of potassium and lactate levels by $94 \%$ and $99 \%{ }^{25}$ and restoration of sodium levels, from 93 to $140 \mathrm{mmol} / \mathrm{L}$, have been reported. ${ }^{26}$

To our knowledge, this is the first study evaluating the effect of mechanical rinsing on phthalate concentration in RBCUs. By employing a cell salvage device, we found a reduction of both DEHP (53\%) and MEHP (87\%) in washed RBCUs, which implies that the exposure of these contaminants to patients can be reduced effectively. The impact was more pronounced with higher initial phthalate concentrations prior washing. However, a considerable amount of plasticizers may remain, which should be avoided particularly in highly vulnerable patients, for example, neonates.

Particularly in the treatment of premature neonates and newborns, the long-standing effect of phthalates on fertility should not be underestimated, and the awareness of a potential phthalate intoxication should be raised. ${ }^{9,27}$ The recommended maximum TDI of DEHP is $50 \mu \mathrm{g} / \mathrm{kg}$ body weight. ${ }^{9}$ By the combination of a short storage time (maximum 7 days) and mechanical rinsing, the exceedance of the recommended TDI can be reduced remarkably from 14.8 -fold to 2.3 -fold when administering a quantity of $100 \mathrm{~mL}$ of RBCs in a $5-\mathrm{kg}$ infant. As a consequence of these results, it is worth considering whether it would be pertinent to use blood products that have been rinsed by mechanical means on the day of surgery, or indeed within a few hours of use, particularly for neonates and infants who are expected to need packed red cell transfusions:

'The U.S. Food and Drug Administration (FDA) and European Commission advocate abolition of DEHP for all products in contact with high risk groups such as children before the onset of puberty, and suggest that alternative materials without any relevant detrimental effects be used, if they exist, in order to minimize health risks from products containing DEHP'.27,28

There are many alternatives to replace the common plasticizers in RBCUs. The European Commission specifies tri-(octyl) trimellilate (TOTM) as an alternative plasticizer for stored RBCs, which is included in the 'List of Additives' in the Synoptic document no. 7 of the European Commission ref. no $94800 .{ }^{9,29}$ It is less harmful for patients, staff and the environment. Moreover, recent studies demonstrated the considerably slower migration of TOTM into blood compared to DEHP. ${ }^{2,19,30}$ Other good alternatives like silicone and polyurethane, which are widely used for platelet and plasma storage bags, have critical financial implications for producers of whole blood bags. ${ }^{31}$ RBCUs stored in bags plasticized with 1,2-cyclohexane dicarboxylic acid diisononyl ester (DINCH) could be an alternative to bags releasing DEHP as well..$^{32}$ However, concerning the membrane stabilizing effect of DEHP, these alternatives such as poly-(ethylene-co-ethyl acrylate) (EEA) do not currently show the same quality regarding the stability of the erythrocyte membrane during longterm storage. ${ }^{31,33-35}$

\section{Limitations}

Measurements of unwashed and washed RBCUs were consecutive in different settings. Hence, equal groups would have been more conclusive. 


\section{Conclusion}

In conclusion, this study demonstrates that the burden of phthalate concentrations in RBCUs can be effectively reduced by shortening the storage time and by mechanical rinsing. The legal regulations of the European Commission for minimizing the risks from medical products containing DEHP and other plasticizers demand the relegation of risks from the use of medical products containing DEHP. ${ }^{9}$ Cell salvage of RBCUs allows one to fulfil these demands. Alternatively, manufacturers of medical devices could rethink producing phthalate-free single-use materials in the long term.

\section{Acknowledgements}

The authors thank Mr Johannes Müller for the necessary laboratory work. They also thank Carole Hamilton for proofreading their paper as a native English speaker. This study was completed in fulfilment of the requirement for cumulative doctoral studies for the degree 'Dr. rer. biol. hum'.

\section{Declaration of Conflicting Interests}

The author(s) declared no potential conflicts of interest with respect to the research, authorship and/or publication of this article.

\section{Funding}

The author(s) received no financial support for the research, authorship and/or publication of this article.

\section{References}

1. Buchta C, Bittner C, Heinzl H, et al. Transfusion-related exposure to the plasticizer di(2-ethylhexyl)phthalate in patients receiving plateletpheresis concentrates. Transfusion 2005; 45: 798-802.

2. Eckert E, Münch F, Göen T, et al. Comparative study on the migration of di-2-ethylhexyl phthalate (DEHP) and tri-2-ethylhexyl trimellitate (TOTM) into blood from PVC tubing material of a heart-lung machine. Chemosphere 2016; 145: 10-16.

3. Hildenbrand SL, Lehmann HD, Wodarz R, et al. PVCplasticizer DEHP in medical products: do thin coatings really reduce DEHP leaching into blood. Perfusion 2005; 20: 351-357.

4. Kambia K, Dine T, Azar R, et al. Comparative study of the leachability of di(2-ethylhexyl) phthalate and tri(2ethylhexyl) trimellitate from haemodialysis tubing. Int $J$ Pharm 2001; 229: 139-146.

5. Leuenberger N, Barras L, Nicoli R, et al. Urinary di(2-ethylhexyl) phthalate metabolites for detecting transfusion of autologous blood stored in plasticizer-free bags. Transfusion 2016; 56: 571-578.

6. Koch HM, Bolt HM, Preuss R, et al. Intravenous exposure to di(2-ethylhexyl)phthalate (DEHP): metabolites of DEHP in urine after a voluntary platelet donation. Arch Toxicol 2005; 79: 689-693.
7. Rael LT, Bar-Or R, Ambruso DR, et al. Phthalate esters used as plasticizers in packed red blood cell storage bags may lead to progressive toxin exposure and the release of pro-inflammatory cytokines. Oxid Med Cell Longev 2009; 2: $166-171$.

8. Jaeger RJ, Rubin RJ. Migration of a phthalate ester plasticizer from polyvinyl chloride blood bags into stored human blood and its localization in human tissues. $N$ Engl J Med 1972; 287: 1114-1118.

9. European-Commission. The safety of medical devices containing DEHP-plasticized PVC or other plasticizers on neonates and other groups possibly at risk, https:// ec.europa.eu/health/scientific_committees/emerging/ docs/scenihr_o_047.pdf (2016, accessed 19 April 2019).

10. Rowdhwal SSS, Chen J. Toxic effects of di-2-ethylhexyl phthalate: an overview. Biomed Res Int 2018; 2018: 1750368.

11. Rusyn I, Peters JM, Cunningham ML. Modes of action and species-specific effects of di-(2-ethylhexyl)phthalate in the liver. Crit Rev Toxicol 2006; 36: 459-479.

12. European Union. Commission regulation (EU) 2015/326, https://eur-lex.europa.eu/eli/reg/2015/326/oj (2015, accessed 19 April 2019).

13. European Union. Directive 2005/84/EC, https://eur-lex. europa.eu/LexUriServ/LexUriServ.do?uri=OJ:L:2005:34 4:0040:0043:en:PDF (2005, accessed 3 April 2019).

14. Amand T, Pincemail J, Blaffart F, et al. Levels of inflammatory markers in the blood processed by autotransfusion devices during cardiac surgery associated with cardiopulmonary bypass circuit. Perfusion 2002; 17: 117-123.

15. Melo A, Serrick CJ, Scholz M, et al. Quality of red blood cells using the Dideco Electa autotransfusion device. $J$ Extra Corpor Technol 2005; 37: 58-59.

16. German Medical Association Paul Ehrlich Institute. Guidelines for the collection of blood and blood components and the use of blood products (hemotherapy) (in German). https://www.bundesanzeiger.de/ ebanzwww/wexsservlet?page.navid=to_bookmark official\&bookmark_id=aAYgqkUFS8tt0YXUhbo (2017, accessed 19 April 2019).

17. Höllerer C, Müller J, Göen T, et al. Isomeric separation and quantitation of di-(2-ethylhexyl) trimellitates and mono-(2-ethylhexyl) trimellitates in blood by LC-MS/ MS. J Chromatogr B Analyt Technol Biomed Life Sci 2017; 1061-1062: 153-162.

18. Eckert E, Müller J, Göen T. Simultaneous determination of polyvinylchloride plasticizers di(2-ethylhexyl) phthalate and tri(2-ethylhexyl) trimellitate and its degradation products in blood by liquid chromatographytandem mass spectrometry. J Chromatogr A 2015; 1410: 173-180.

19. Münch F, Höllerer C, Klapproth A, et al. Effect of phospholipid coating on the migration of plasticizers from PVC tubes. Chemosphere 2018; 202: 742-749.

20. Testai E, Hartemann P, Rastogi SC, et al. The safety of medical devices containing DEHP plasticized PVC or other plasticizers on neonates and other groups possibly at risk (2015 update). Regul Toxicol Pharmacol 2016; 76: 209-210. 
21. Hothorn T, Hornik K, Zeileis A. Unbiased recursive partitioning: a conditional inference framework. Journal of Computational and Graphical Statistics 2006; 15: 651-674.

22. Rock G, Labow RS, Tocchi M. Distribution of di(2-ethylhexyl) phthalate and products in blood and blood components. Environ Health Perspect 1986; 65: 309-316.

23. Inoue $\mathrm{K}$, Kawaguchi M, Yamanaka R, et al. Evaluation and analysis of exposure levels of $\operatorname{di}(2$-ethylhexyl) phthalate from blood bags. Clin Chim Acta 2005; 358: 159-166.

24. Strauss RGST, McCullough J, Snyder EL, et al. Red blood cell transfusions for neonates and infants. 5th ed. Oxford: Wiley-Blackwell, pp. 535-541, 2016.

25. Westphal-Varghese B, Erren M, Westphal $M$, et al. Processing of stored packed red blood cells using autotransfusion devices decreases potassium and microaggregates: a prospective, randomized, single-blinded in vitro study. Transfus Med 2007; 17: 89-95.

26. Read MS, Coles P, Pomeroy M, et al. Conditioning outof-date bank-stored red blood cells using a cell-saver auto-transfusion device: effects on numbers of red cells and quality of suspension fluid. Anaesthesia 2014; 69: 1206-1213.

27. FDA public health notification: PVC devices containing the plasticizer DEHP, http://www.statcorpmedical.com/avactis-images/u/06-09\%20FDA\%20Public\%20Health\%20 Notification-\%20PVC\%20Devices\%20Containing\%20 the\%20Plasticizer\%20DEHP.pdf (2002, accessed 19 April 2019).

28. European-Commission. Medical devices containing DEHP plasticised PVC; Neonates and other groups possibly at risk from DEHP toxicity, http://ec.europa.eu/ health/ph_risk/committees/scmp/documents/out43_ en.pdf (2002, accessed 19 April 2019).

29. European-Commission. Synoptic document - Provisional list of monomers and additives notified to European commission as substances which may be used in the manufacture of plastics and coatings intended to come into contact with foodstuffs, http://www.contactalimentaire.com/ fileadmin/ImageFichier_Archive/contact_alimentaire/ Fichiers_Documents/Avis_de_AESA/synoptic_doc_ en_-_version_June_2005.pdf (2005, accessed 19 April 2019).

30. Klapproth A, Schärli M, Göen T, et al. Migration of additives (DEHP \& TOTM) from Ph.i.s.i.o. ${ }^{\circledR}$-coated PVC materials in medical devices (in German). Kardiotechnik 2017; 26: 3-11.

31. Prowse CV, de Korte D, Hess JR, et al. Commercially available blood storage containers. Vox Sang 2014; 106: $1-13$.

32. Serrano K, Levin E, Chen D, et al. An investigation of red blood cell concentrate quality during storage in paediatric-sized polyvinylchloride bags plasticized with alternatives to di-2-ethylhexyl phthalate (DEHP). Vox Sang 2016; 110: 227-235.

33. Horowitz B, Stryker MH, Waldman AA, et al. Stabilization of red blood cells by the plasticizer, diethylhexylphthalate. Vox Sang 1985; 48: 150-155.

34. Rock G, Tocchi M, Ganz PR, et al. Incorporation of plasticizer into red cells during storage. Transfusion 1984; 24 : 493-498.

35. Simmchen J, Ventura R, Segura J. Progress in the removal of di-[2-ethylhexyl]-phthalate as plasticizer in blood bags. Transfus Med Rev 2012; 26: 27-37. 